\title{
Modeling Flow in Disordered Packed Beds from Pore-Scale Fluid Mechanics
}

\author{
Karsten E. Thompson and H. Scott Fogler \\ Dept. of Chemical Engineering, The University of Michigan, Ann Arbor, MI 48109
}

\begin{abstract}
Network models are an effective means of incorporating pore-scale heterogeneity into flow models of porous materials. The drawback to these models used to be the inability to obtain quantitative macroscopic parameters representing larger (experimental-scale) media. However, recently developed modeling techniques, combined with more widely available computational resources, make the simulation of macroscopic parameters from a network approach viable. A network model for the slow flow of an incompressible fluid in disordered packed beds is presented. Fundamental fluid mechanics equations are solved at the pore scale and then translated to macroscopic behavior using a network approach. The results reproduce experimental permeabilities and show excellent quantitative fits to residence time distributions for mechanical dispersion in real beds. Simulations of the RTD are of special interest, because they are definitive links between pore-scale flow behavior and macroscopic responses.
\end{abstract}

\section{Introduction}

Models for flow in porous media occur over a vast range of length scales, and the appropriateness of any one usually depends on the length scale over which the phenomena of interest operate. Moving from large to small length scales, one can delineate three categories: models that account for macroscopic heterogeneity; continuum models of homogeneous media; and at the smallest scale, pore-level models. Models of the first type account for the variation in physical properties on a scale much larger than the pore dimensions. They are used for studying oil production, groundwater transport, and contaminant migration, all of which are situations where macroscopic heterogeneity contributes strongly to transport processes. On a smaller length scale, media are treated as a macroscopically uniform continuum. This assumption is often good, and it allows physical parameters such as permeability, saturation, capillary pressure, and superficial velocity to be written as smooth functions, even though at the pore level quantities such as saturation or capillary pressure are discrete, and the fluid velocity is highly variable.

K. E. Thompson is presently at the Department of Chemical Engineering, Louisiana State University, Baton Rouge, LA 70803.
Pore-level models include the smallest length scales that affect the morphology of a medium. They sometimes are limited by their inability to effectively reproduce macroscopic quantities, but this limitation has not diminished their importance. In certain situations, such as carbonate acidizing, macroscopic phenomena depend so strongly on microscopic effects that continuum models fail. Furthermore, although quantities such as permeability are highly effective in describing flow in a homogeneous medium, they ultimately depend on fluid dynamics at the pore level. Hence, the desire to understand underlying fundamentals dictates the need for modeling at this small scale.

In this article we present a series of recent developments and new techniques that are combined into a quantitative model for flow through a packed bed. The model accounts for pore-scale heterogeneity using a network approach. However, the network development includes both a complete description of the bed and a fundamental description of pore-scale fluid mechanics. The significance of the resulting model is its ability to generate quantitative macroscopic parameters from the most basic knowledge of a medium's morphology, thus allowing pore-scale and multiple-pore heterogeneity to be accounted for automatically. 


\section{Pore-level Modeling}

Pore-level models include morphologic, chemical, or probabilistic parameters that are unique to this length scale. Capillary-bundle models are the simplest type. They incorporate flow variation, but in neglecting the effects of interconnectivity and tortuosity they are useful primarily as conceptual tools. Volume averaging is a widely used technique in which a macroscopic momentum equation is derived from the Navier-Stokes equations averaged over a small representative elementary volume. During the averaging process, hydrodynamic information from the pore scale is retained, but it is reintroduced in the form of unknowns that must be determined experimentally or derived for very simple pore structures (e.g., Kaviany, 1991). Network models use a series of interconnected nodes and bonds with distributed sizes. By assigning flow resistances to the bonds, a numerical solution for the pressures (and hence velocities) within the network is obtained.

\section{Network modeling techniques}

While the idealization of a pore space as a network of nodes and bonds requires simplifying assumptions, network models have the distinct advantage of accounting for pore-level heterogeneity and interconnectivity. Traditionally, their limitations have been network size (controlled of course by computer speed and storage) and the inability to produce quantitative, dimensional results. Comparisons to data that do exist usually rely on arbitrary parameters or dimensional arguments to correlate the flow in individual bonds to a spatially averaged flux (or Darcy velocity).

The distinction between various network models lies primarily in the choice of lattice arrangement, node and bond geometry, and in the specific solution algorithm used. The parameters that dictate a network's geometry are its spatial dimension (i.e., 2-D or 3-D), grid pattern (which may be regular or irregular), bond-size distribution, and coordination number (the value and whether it is constant). The most common methods for introducing randomness into the lattice structure are to use Voronoi and Delaunay tessellations (e.g., Jerauld et al., 1984a,b; Mason and Mellor, 1991; Blunt and King, 1990), although other methods have also been proposed (Constantinides and Payatakes, 1989). Guidelines for network structure have been developed through comparisons of regular and irregular networks (Jerauld et al., 1984a,b) and by topology of actual media (Dullien, 1975; Koplik et al., 1984). However, the use of an assumed pore-size distribution to decorate a regular network grid remains a common method.

The solution method used most widely in petroleum-related research was proposed originally by Fatt (1956) and consists of the numerical solution for fluid pressure at every node. To employ this method, one sets up a governing system of linear equations by applying a fluid-conservation equation around each node and assigning a flow conductivity to every bond in the network. Somewhat different methods are used for percolation theory, which lacks certain dynamic effects, but is a useful tool for relating network structure to conduction and saturation. It has been used to study interconnectivity and network geometry (Jerauld et al., 1984a,b), the physical structure of real media (Mason and Mellor, 1991), and the origin of multiphase permeabilities (Heiba et al., 1992; Larson et al., 1981). Effective medium theory is also a lattice-based approach (Koplik, 1982), but the pore-scale description of the network is lost during the averaging process.

\section{Fluid transport in network models}

Network modeling has been used consistently over the last three decades. Its evolution reflects advances in theory and techniques, but equally the vast changes in computational power. Early contributions by Dullien (1975) and later by Koplik et al. (1984) aimed to bring realistic pore structure into their models by using photomicrography of actual media to map out networks. Other efforts have resulted in more complex descriptions of fluid behavior, such as a creeping flow solution in circular pore bodies (Koplik, 1982) and the use of bonds with sinusoidally varying cross sections (Lin and Slattery, 1982; Dias and Payatakes, 1986; Hopkins and Ng, 1986). Mohanty and Salter (1982) used a relatively simple cubic network to reproduce a number of macroscopic experimental results and were the first to incorporate many detailed multiphase effects.

The dispersion of solute during flow in porous media is one of the topics to which network modeling has contributed most because it is a macroscopic phenomenon dominated by pore-scale effects. The methods for modeling dispersion are generally statistical; the convective and/or diffusive movement of a very large number of particles is analyzed in order to obtain information about either spatial displacements or particle transit times. Examples include the work of Sahimi et al. (1983, 1986), who used Monte Carlo simulations on 2and 3-D lattices to demonstrate the macroscopically diffusive behavior of tracer dispersion, and Sorbie and Clifford (1991), who used network models to study dispersion over a wide range of Peclet numbers. Other important phenomena that have been explained using network modeling are the masstransfer-limited dissolution of porous media (e.g., the acidizing of carbonate formations for oil production) (Hoefner and Fogler, 1988) and the deep-bed filtration and straining of colloidal particles in porous media (e.g., Rege and Fogler, 1988, 1987). Both of these phenomena, while absolutely dependent on pore-level events, cause substantial macroscopic responses.

Despite the success of network models for describing pore-scale phenomena, a more elusive trait is the direct simulation of macroscopic or averaged parameters. This scale-up requires large enough networks to provide statistically significant information, and the simplifying assumptions inherent to any network are compatible with the simulated process. Blunt and King (1991) address the scale-up of macroscopic parameters in 2- and 3-D network models, investigating both capillary-dominated and viscous-dominated immiscible displacements in order to relate relative permeability and fractional flow to saturation. While large networks were used for these studies, topologic limitations are a problem.

\section{Physically representative network model}

The most significant recent advance in network modeling is the work of Bryant and Blunt (1992) and Bryant et al. (1993a,b), who developed a physically representative network 
model. The flow distribution is obtained in a standard manner (solving a system of linear fluid-conservation equations), but the network development is unique. A 3-D packed bed (Bryant et al. use a real random packing with known sphere coordinates) is mapped onto a network using a Delaunay tessellation, which in three dimensions forms a space-filling (but with no overlap) array of tetrahedrons, each having four vertices at sphere centers. It enables a completely described continuum pore space to be discretized into a well-defined framework without sacrificing topographic information, and it lends easily to the summation of pore properties such as porosity, surface area, and saturation to obtain overall values for an entire packed bed. It should be noted that Delaunay tessellations were used previously for pore space analysis, but in applications different than those discussed in this article (Mason, 1967; Mellor, 1989; Chan and Ng, 1988). Bryant et al. (1993b) used an analysis of the random packing to show that the assumption of constant path length in a network is invalid, as is the assumption of randomly correlated conductivities. The original packing was used to simulate a consolidated sandstone by introducing compaction in one direction (i.e., compression of one Cartesian axis) and cementation (achieved by increasing the radii of the spheres while maintaining the coordinates of their centers). Favorable agreement with permeability was found when compared to Fontainebleau sandstone, which has a narrow distribution of grain size, but a wide variation in the amount of cementation, resulting in permeabilities that range over three orders of magnitude.

While the physically representative network model just discussed is an important advance in network modeling technology, the network structure is limited by its connection to the single real packing, and significant assumptions are made regarding pore-scale fluid conductivity. The work reported in this article was performed in order to develop a general model for fluid and solute transport in disordered packed beds. The goals included the following: the simulation of an arbitrary packing arrangement; the use of more rigorous fluid mechanics at the pore scale (thus lending credence to simulations of phenomena that depend on pore-scale events); the incorporation of this information into a model capable of simulating macroscopic phenomena for beds with essentially unlimited heterogeneity.

\section{Model Development}

\section{Overview}

The bed is created by a computer algorithm that simulates the random packing of spheres into a specified volume. A Delaunay tessellation is used to transform the bed into a network in the manner described by Bryant et al. (1993a,b). Although the resulting Delaunay tetrahedrons are used to set up the network fluid conservation equations, all microscopic fluid transport equations are solved by examining the locally bounding spheres. The result is a distinct link between porelevel phenomena and macroscopic behavior in the bed. Five steps in the modeling process are described in the following sections:

1. Simulation of the packed bed

2. Delaunay tessellation

3. Solution of fluid transport equations at the pore level
4. Solution of the overall pressure and flow profile

5. Modeling solute transport and residence time distributions.

As the model development will show, this methodology is superior to typical network modeling techniques because disorder and heterogeneity are introduced into the structure of the packed bed rather than by randomly assigning size distributions onto the network itself. Furthermore, the physical description of the bed is not compromised during the transformation into a network problem.

\section{Computer-simulated packed bed}

For the tessellation and the network algorithm to be implemented, a complete description of a packed bed is required (consisting of an array containing the $x, y$ and $z$ coordinates and the radius of every sphere in the pack). The quantity and detail of this information suggest the use of a computersimulated packed bed so that one can quickly determine what is all but impossible to obtain experimentally.

The input parameters dictating the physical properties of the simulated bed are chosen in a manner analogous to specifying the dimensions of a real packing. The bed is initialized by selecting the mean sphere diameter, the size distribution (which in the simplest case requires the standard deviation from the mean diameter), the cross-sectional area of the pack (typically defined by either the dimensions of a rectangular base or the radius of a circular base), and finally the number of particles to be placed in the bed. The latter quantity can be replaced by the length of the pack, but the number of particles is often more desirable because it dictates the number of pores and hence directly affects computation speed.

The basic algorithm used to create a sphere pack is taken from the literature (Visscher and Bolsterli, 1972). It is a drop-and-roll-type algorithm that results in a pack being built sphere-by-sphere from the base. The base spheres are placed on a lattice of preset dimensions, and then disturbed spatially in order to prevent degeneracies from occurring during the tessellation process. The packing process proceeds by selecting a sphere from the designated size distribution and selecting a random $x, y$ coordinate above the bed from which the sphere is released. The first point of contact with the bed is calculated based on a $z$-direction fall, from which a series of rolls are simulated, allowing the dropped sphere to settle onto the packed bed. The rolls are complete when the dropped sphere reaches a stable gravitational minimum, which means that it is in contact with at least three spheres and any further movement would first require an upward roll. Qualitatively, this condition can be envisioned as the dropped sphere coming to rest on a triangle formed by three other spheres in the bed. In large beds, four-sphere contacts occur occasionally (within the accuracy of the real-number calculations).

The porosity of the beds created by the computer simulation is fairly consistent, ranging from 0.38 to 0.42 for both uniform and nonuniform sphere distributions. The algorithm can be modified to obtain other porosities by performing a number of drops for each sphere and selecting the final result that most closely conforms to the desired property (for instance, low porosity). Other constraints or additions to the algorithm can be used to create specific or unusual packing arrangements, as shown below. During the creation of mod- 
erate to large-sized packs, unusual geometric configurations can arise, which can be dealt with by modifications to the basic packing algorithm. These changes have been made so that the current program can rapidly create packs in excess of 20,000 spheres if the standard deviation in sphere diameter is less than 0.25 times the mean. Broader size distributions can be obtained if one is willing to discard the drop attempts that cause problems. Also, other algorithms are available in the literature that can be incorporated into this section of the model (see, for instance, Yang et al., 1996).

\section{Delaunay tessellation}

A sphere-by-sphere ordering of a packed bed enables a complete physical description to be obtained for any local region of pore space. However, a network algorithm to simulate flow through the pack requires that the pore space be divided into discrete elemerits (pores) connected to one another in a well-defined manner. The Delaunay tessellation is appropriate because it divides spheres into nearest-neighbor groups of four, and each Delaunay cell closely resembles the general description of a pore.

The four vertices of a Delaunay tetrahedron are centers of spheres in the pack. A Delaunay cell can be defined uniquely by examining the circumsphere defined by the four vertices; the definition of a Delaunay tetrahedron requires that this circumsphere encompass no other sphere centers from the pack (Bowyer, 1981; Watson, 1981). A first-order degeneracy occurs when a fifth sphere center lies on the circumsphere. It is rare in a disordered pack, but it does occur and can be resolved by dividing the five spheres into two tetrahedrons that share a face. The dimensions of these two tetrahedrons typically dictate the way in which this division occurs (for instance, one may attempt to obtain faces similar to equilateral triangles).

Figure 1 shows four spheres arranged to form a tetrahedron. Imagine, in Figure 1a, the bottom three spheres resting on a horizontal surface with the fourth sphere placed on this triangular base. From this mental picture, it should be evident that a region of space exists in the center of this group of four spheres. Access to this space is through any of four constrictions that are created where three spheres meet. This picture leads naturally to the definition of a pore space: a central void with a number of distinct constrictions connecting the void to the remaining pore space. Figure 1b shows the tetrahedron that might be formed by these spheres, showing how the sphere surfaces cut through the tetrahedron faces.

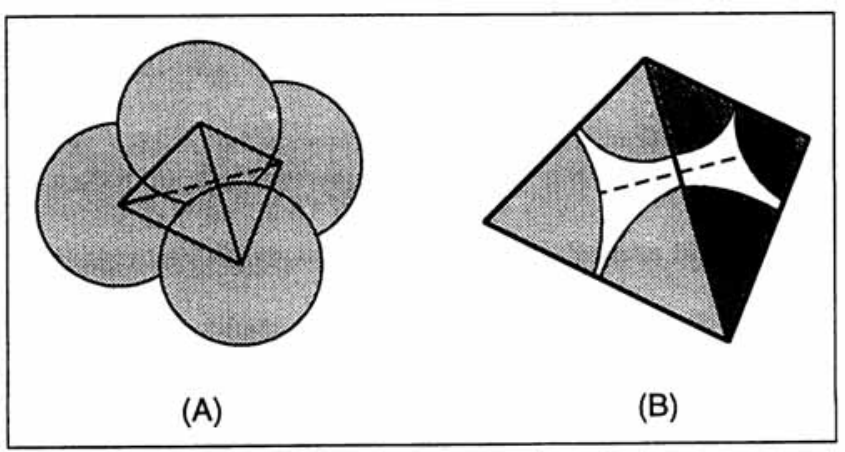

Figure 1. (a) Four-sphere pore; (b) network analog.
Because the vertices are at sphere centers, the void projected onto each of the four faces is necessarily the smallest constriction that will be encountered when traversing a passage into the central pore. Hence, the area projected onto a tetrahedron's face represents a pore throat in the network analog. It should be noted that despite a group of four spheres satisfying the nearest-neighbor tessellation criterion, they do not necessarily touch (a result of contact with surrounding spheres). This fact introduces significant variability to the shape of a pore throat.

The algorithm to perform a tessellation is, in principle, simple. A seed tetrahedron is found, the search for which need not be efficient because it occurs only once. This tetrahedron has four faces, each of which must be shared with another tetrahedron (unless the face is a boundary). The algorithm proceeds from a seed face that has an unknown neighbor, searching to find a fourth vertex to complete a new tetrahedron. For a nondegenerate face, this fourth point is unique. As tetrahedrons are added to the tessellation, additional seed faces are created. The search continues until no seed faces are available, which indicates a completed tessellation. During the tessellation process, the boundary for allowed tetrahedrons lies a few sphere layers inside the edge of the packed bed. This strategy ensures that the edge tessellations are uniform with respect to the rest of the bed. The nonconducting edge faces then define the final network boundary. It is of interest to note that the Delaunay tessellation is the geometric dual of the Voronoi tessellation (Watson, 1981). Each Voronoi polyhedron is associated with a single vertex (a sphere), and the volume within the polyhedron is nearer its associated vertex than any other.

The Delaunay tessellation is used not only to define individual pores, but also for the equally important role of defining pore interconnectivity. Because the tessellation results in a space-filling aggregate of tetrahedrons (Watson, 1981), any nonedge face must border a neighbor tetrahedron (further illustrating the role of the face as a connecting throat). When used in this way, the completed tessellation must provide the following information for each tetrahedron: its four vertex spheres and the four other tetrahedrons with which it shares a face. Total storage for the completed description of the bed requires a real-number array for the sphere positions and radii, which has dimensions (number of spheres $\times 4$ ), plus an integer array (for the tessellation) containing the numbers of vertex spheres and neighboring pores, which has dimensions (number of tetrahedrons $\times 8$ ). It is again emphasized that, because the information from the tessellation points to sphere positions and radii, no morphologic information is lost during the network transformation. Two other notes are made here. First, the position array is stored in dimensionless form in order to allow a given arrangement of spheres to be used to simulate a pack of any mean sphere size. Second, because large packs are used in the simulations, wraparound boundary conditions are not used. Pores at the edge of the pack have at least one nonconductive face, which is representative of real packed beds.

While the flow calculations within discrete pores are the most important aspect of the modeling, the Delaunay tessellation provides a rapid and simple means for calculating other physical quantities. Porosity is the most obvious: the porosity of the entire bed is calculated by summing the void volume 
and total volume of each tetrahedron. Because spheres at the edge of the tessellated region are only partially contained within tetrahedrons, this method is superior to summing the volume of all spheres to calculate porosity. As shown in Eq. 1 , the summation also provides an accurate method for determining the average cross-sectional area of the pack (area varies with bed length even for cylindrical beds because the network boundary is formed by irregular tetrahedrons):

$$
A_{c s}=\frac{V_{\text {bed }}}{L}=\frac{\sum_{\text {pores }} V_{\text {tet }}}{L} .
$$

Similar summations give exact values for saturation, surface area, and concentration.

In using the Delaunay tessellation, the network is automatically restricted to a coordination number of four. While this restriction affects the calculation of percolation properties, it does not adversely affect the simulation of single-phase fluid transport because the tessellation is primarily a tool to organize the pore space into discrete elements. In situations where the local topology does not conform easily to single-pore divisions by the tessellation process, void spaces are defined using more than one tetrahedron. An example is a situation where spheres from a bed are removed to create a single large void (a vug in petroleum literature). While this large void may be discretized by multiple tetrahedrons, their common faces will contain unusually large projected voids, which will have low fluid resistances. Local transport will be dominated by these regions of high conductivity, as would be expected in a true heterogeneity of this type.

\section{Single-phase flow in the network}

The most significant challenge to modeling flow through the packed bed is to generate an accurate description of flow at the pore level and to scale-up this information to create a global picture of fluid and mass transport. We begin by examining the system of equations to be solved for flow through the network. As discussed earlier, the final flow solution is obtained from a linear matrix equation representing fluid conservation at each pore. To obtain this equation one writes a balance on the net volumetric flow rate in each interior tetrahedron, $i$,

$$
q_{\mathrm{net}}=0=\sum_{j=1,4}\left[\left(p_{j}-p_{i}\right) g_{i, j}\right]
$$

where $g_{i, j}$ is a fluid conductivity term (equal to the ratio of volumetric flow rate and pressure drop across a pore throat). The subscript $j$, which denotes the four neighboring pores, illustrates the role of the tessellation in mapping out the interconnectivity of the pack. The matrix equation is obtained by rearranging Eq. 2 for $p_{i}$, the pressure in each pore. The outlet pressure is typically set to zero, meaning that for pores bordering the outlet, the sum of the flow rates will have an unknown but nonzero value. The analogous situation is true if the inlet pressure is constant. Alternatively, a constantinjection-rate model is obtained by adding a single constraining equation to the matrix that represents the sum over all inlet pores:

$$
Q=\sum_{j=\text { inlet }}\left(p_{\text {inlet }}-p_{i}\right) g_{i, j}
$$

The system of linear equations is large and sparse, hence is solved using the Gauss-Seidel method with overrelaxation. (Use of the tessellation eliminates the need to set up the entire coefficient matrix; only the nonzero terms are stored.)

\section{Pore-scale fluid mechanics}

When using Eq. 2, the Delaunay tessellation specifies the four pores $j$ that neighbor a given pore $i$. One still requires numerical values for the conductivities between any two pores (i.e., the elements $g_{i, j}$ in the coefficient matrix). These conductivities depend on local topography, so they are obtained by reverting back to the original sphere-pack coordinates in order to describe flow in the neighborhood of the bounding spheres.

For single-phase laminar flow, a throat conductivity typically depends on the fluid viscosity and throat geometry, and for simple geometries an equation can be derived analytically. Figure 2, however, shows the cross sections of four different types of throats taken from a computer-generated packed bed. The geometries of these pore channels present three distinct problems for modeling microscopic fluid flow. First, the cross sections of the ducts are somewhat arbitrary, defined by the dimensions of the triangular faces and the radii of the bounding spheres. Second, as discussed before, the spheres do not necessarily touch, meaning that the ducts may not be completely enclosed. Third, because the bounding surfaces are spheres, the cross sections of the ducts converge and diverge (in a direction perpendicular to the page in Figure 2).
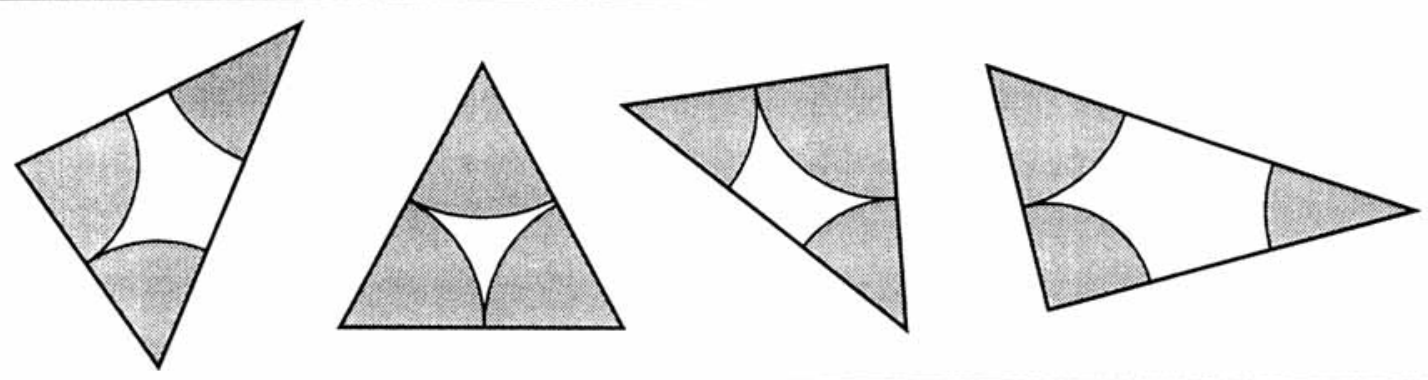

Figure 2. Typical tetrahedron-face geometries in a computer-generated random packing of spheres. 
Since analytical solutions for the desired flow profiles are not available, the possible methods for obtaining the conductivity matrix are to use a general approximation, an empirical formula, or a numerical solution. General approximations that use terms such as the wetted perimeter and effective radius do not have sufficient basis. Semiempirical equations are available in the literature for a number of situations, the most applicable being those by Yilmaz (1990), which give pressure drops for various duct geometries. However, these and other similar equations are valid for rather specific families of shapes and can give results that deviate significantly when duct cross sections do not conform to the required geometric family. In addition, neither of the preceding options can effectively account for unbounded sections of the perimeter. Hence, the approach taken here is to perform a numerical solution for parallel flow through the appropriate cross section, and to correct the result using a solution for creeping flow through a converging-diverging-shaped channel.

To obtain a relationship between flow rate and pressure drop for parallel flow through an arbitrary shaped duct, the Navier-Stokes equations are simplified to

$$
\frac{\partial^{2} u}{\partial x^{2}}+\frac{\partial^{2} u}{\partial y^{2}}=\frac{1}{\mu} \frac{d p}{d z}
$$

In this equation, $u$ represents the $z$-component velocity, where the duct's cross section lies in the $x-y$ plane. For parallel, incompressible flow, the righthand side of this equation is constant. It is desirable to obtain a dimensionless solution so that it can be applied to a throat of specified geometry regardless of its size. Dedimensionalization of Eq. 4 is performed by defining the following characteristic dimensions:

$$
\begin{aligned}
\text { length } & =r_{1} \\
\text { force } & =(d p / d z) / r_{1}^{3} \\
\text { time } & =\mu /\left[(d p / d z) r_{1}\right]
\end{aligned}
$$

The definition for length comes from the geometry of the system (it is the radius of one of the bounding spheres) while the definitions for force and time can be obtained by examining Eq. 4. These definitions result in four dimensionless variables.

$$
\begin{gathered}
x^{*}=x / r_{1} ; \quad y^{*}=y / r_{1} ; \quad u^{*}=u \mu /\left[r_{1}^{2}(d p / d z)\right] \\
Q^{*}=Q \mu /\left[r_{1}^{4}(d p / d z)\right]
\end{gathered}
$$

The dimensionless flow rate is used later. Substituting $x, y$, and $u$ into $\mathrm{Eq} .4$ gives

$$
\frac{\partial^{2} u^{*}}{\partial x^{* 2}}+\frac{\partial^{2} u^{*}}{\partial y^{* 2}}=1
$$

This dimensionless form of Poisson's equation, like Eq. 4, describes parallel flow through any arbitrary duct, such as those shown in Figure 2. Because of the unusual geometries of the cross sections, this equation must, in general, be solved numerically.
The equation is solved using the finite-element method. Due to the large number of solutions that must be obtained for a given bed, a general finite-element routine was adapted specifically for the tetrahedron geometries encountered and built directly into the overall code. Solution of Eq. 5 gives the velocity as a function of position on the face, as shown by the contours in Figure 3. Also shown in Figure 3 is the difference between assuming a zero-velocity vs. a zero-flux boundary condition at the gaps between spheres. It is apparent from Figure 3 and from the values of $Q^{*}$ that the zero-velocity assumption assigns an abnormally low conductivity to a long, thin pore throat bordering a gap.

The dimensionless velocity $u^{*}=f\left(x^{*}, y^{*}\right)$ is integrated to determine the dimensionless flow rate defined previously:

$$
Q^{*}=\int_{A} u^{*} d x^{*} d y^{*}
$$

Finally, substituting the definition of $Q^{*}$ and assigning $d p / d x$ $=\Delta P / L$, an expression for $g$ is obtained for a given pore throat,

$$
g=\frac{Q}{\Delta P}=Q^{*}\left(\frac{r_{1}^{4}}{\mu} \frac{\Delta P}{L}\right) \frac{1}{\Delta P}=\frac{Q^{*} r_{1}^{4}}{\mu L}
$$

in which the $i$ and $j$ subscripts have been dropped for clarity. This equation transforms the dimensionless flow rate into the term $g_{i, j}$ needed in Eqs. 2 and 3. For a given bed, it is advantageous to create a file of the dimensionless flow rates, $Q^{*}$,
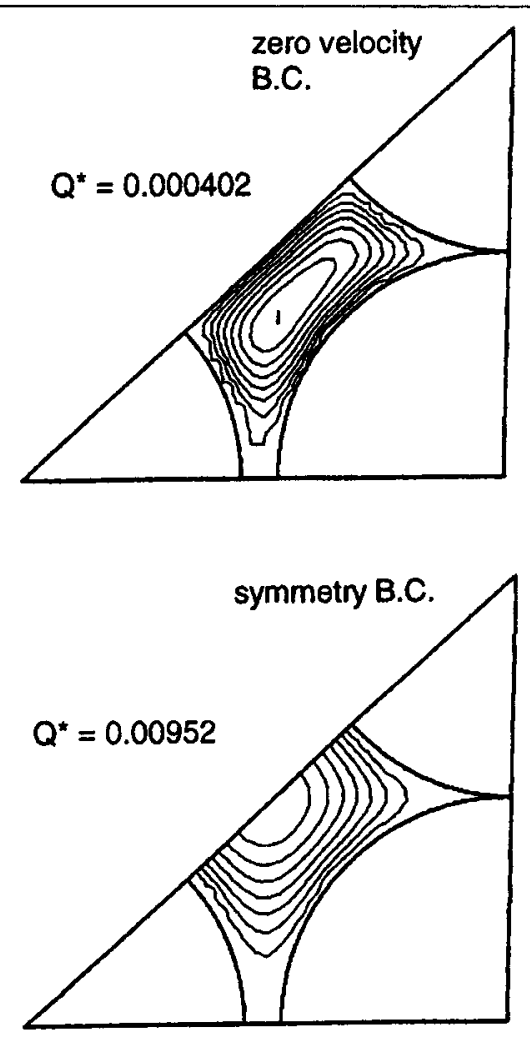

Figure 3. Pore-throat velocity profiles for two different boundary conditions. 
and the dimensionless sphere positions because this information is restricted to a specific bed structure but not to the mean diameter nor to fluid viscosity.

The numerical solution just described provides a solution for parallel flow through an arbitrary duct that may or may not be enclosed by a zero-velocity perimeter, hence satisfying two of the requirements listed previously. The final problem in calculating the fluid resistance terms is that the flow converges and diverges as it passes through the constriction leading into a Delaunay cell. A number of approximations for converging-diverging flow have been introduced into network models (e.g., Lin and Slattery, 1982; Bryant et al, 1993a). In the current work, a solution for creeping flow in a hyperbolic Venturi is used to assess the effect of the convergingdiverging geometry. It was chosen for convenience and because of the appropriateness of its shape, and is introduced as a correction to Eq. 7 .

To map the actual pore throat geometry onto the Venturi, one determines the radius of a tube of circular cross section that has the same resistance to parallel laminar flow, as does the duct used in the finite-element method solution. This effective radius, $r_{\text {eff }}$, becomes the radius of the Venturi's neck, while the length $L$ of the Venturi is equal to the average diameter of the three bounding spheres (see Figure 5). The shape of the hyperbola is chosen to closely resemble the curvature of the bounding spheres. Comparison of the resistance to flow through the Venturi vs. the resistance to parallel flow through the tube of radius, $r_{\text {eff }}$, provides the correction factor to Eq. 7, as shown below.

The solution for flow through a one-sheeted hyperboloid of revolution, as shown in Figure 4, is from Happel and Brenner (1973). It is an axisymmetric problem solved using the stream potential. In oblate spheroidal coordinates, the wall of the Venturi is defined by $h=$ constant for a given $c$ (the constant $h_{0}$ representing the asymptotic angle between the axis of rotation and the wall, and $c$ being the distance from the axis of rotation to the focal circle). The values of these

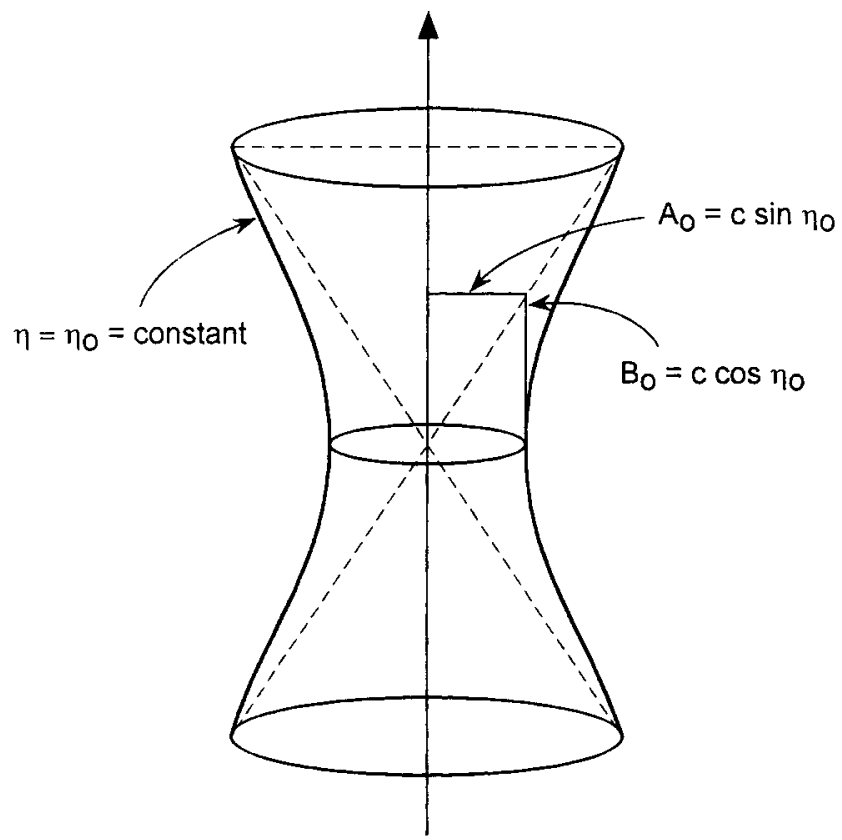

Figure 4. Hyperbolic Venturi.

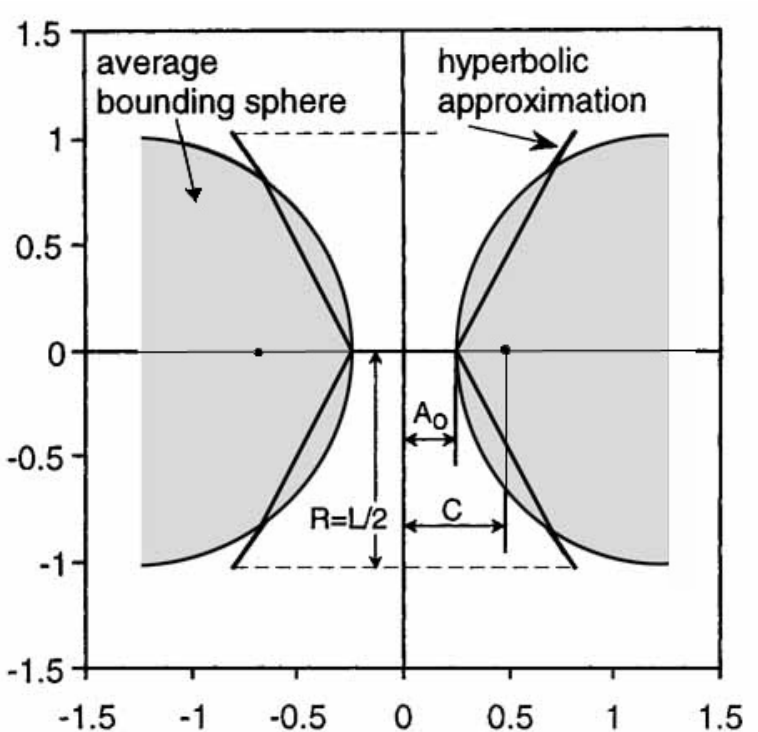

Figure 5. Approximation of pore-throat geometry using the Venturi.

two parameters are dictated by the mapping described in the previous paragraph; specifically, they are defined as

$$
\eta_{o}=\frac{\pi}{2}-\sin ^{-1}\left(\frac{R_{\mathrm{ave}}}{r_{\mathrm{eff}}+R_{\mathrm{ave}}}\right)
$$

and

$$
c=\frac{r_{\text {eff }}}{\sin \eta_{o}} .
$$

where $R_{\text {ave }}$ is the average radius of the bounding spheres (equal to $L / 2$ ). Figure 5 depicts this mapping, comparing two half-circles representing a given $R_{\text {ave }}$ (equal to 1.0 in the graphic) and the hyperbola defined by Eqs. 8 and 9 .

The general equation for the pressure at a point in the Venturi is (Happel and Brenner, 1973)

$$
p=p_{o}-\frac{3 q \mu}{\pi c^{3}\left(1+2 \zeta_{o}\right)\left(1-\zeta_{o}\right)^{2}}\left(\frac{\lambda}{\lambda^{2}+\zeta^{2}}+\tan ^{-1} \lambda\right),
$$

where the reference pressure is taken at the neck of the Venturi, and $\zeta$ and $\lambda$ represent the following change of variables from the oblate spheroidal coordinate system: $\lambda=\sinh \xi$ and $\zeta=\cos \eta$. The pressure drop through the entire Venturi is found by calculating the pressure drop between $z=-R_{\text {ave }}$ and $z=+R_{\text {ave }}$ on the axis of symmetry which, making the appropriate transformations, consists of evaluating Eq. 10 at $\zeta=1$ and $\lambda=\lambda_{R}=R_{\text {ave }} / c$. Rearranging, one can determine a value for the conductivity $g$ of the Venturi,

$$
g_{\text {venturi }}=\frac{q}{\Delta p}=\frac{\pi c^{3}\left(1+2 \zeta_{o}\right)\left(1-\zeta_{o}\right)^{2}}{6 \mu\left(\frac{\lambda_{R}}{\lambda_{R}^{2}+1}+\tan ^{-1} \lambda_{R}\right)}
$$


The conductivity of a capillary having length $L$ and radius $r_{\text {eff }}$ is known from the Hagen-Poiseuille equation (see, for instance, Bird et al., 1960). The ratio of the two conductivities results in the following dimensionless correction factor, which has a value greater than one according to the assumptions made:

$$
\frac{g_{\text {venturi }}}{g_{\text {tube }}}=\frac{4 L\left(1+2 \zeta_{o}\right)\left(1-\zeta_{o}\right)^{2}}{3 r_{\text {eff }}\left(\sin \eta_{o}\right)^{3}\left(\frac{\lambda_{R}}{\lambda_{R}^{2}+1}+\tan ^{-1} \lambda_{R}\right)}
$$

Equation 12 can be used explicitly to adjust the conductivity of the pore throats. In the program, $g_{\text {venturi }} / g_{\text {tube }}$ is calculated only once for each throat and applied directly to the dimensionless flow rate (Eq. 7), which is then stored in the conductivity matrix.

\section{Solute dispersion and residence time distributions}

Solving the previous equations gives the pressure in every pore. From this information, both pore-scale and macroscopic volumetric flow rates can be found. However, modeling dispersion and residence time distributions (RTDs) requires additional assumptions regarding the specific paths and velocities of solute molecules (or tracer particles, in the case of RTDs).

The path that a given tracer particle uses to traverse a model packed bed is determined from probabilistic arguments: once inside a given pore, the exit throat is chosen according to the fraction of flow leaving through that throat. These simple arguments define the overall path taken, but not the time taken to traverse this path. In order to transform microscale volumetric flow rates (which are known from the network solution) to pore residence times without introducing arbitrary scaling parameters, a pore's void space is divided into four weighted volumes. (A pore residence time is defined as the time a fluid element spends in that given pore during its journey through the bed.) Each of these volumes is associated with one of the four faces in the pore, the weights being determined by analyzing the tetrahedral geometry (Thompson, 1996). When both the volumetric flow rate through a face and that face's associated volume are known, one can obtain the average pore residence time associated with face $j$ (the total pore residence time for pore $i$ is found by performing the calculation for the two faces through which the tracer element enters and exits):

$$
\bar{\tau}_{i, j}=\left|v_{i, j} / q_{i, j}\right|
$$

where $v_{i, j}$ is the fraction of pore $i$ 's volume that is associated with face $j$. Subsequently, a probabilistic argument is used to introduce the variation in velocity caused by flow along different streamlines. For an assumed parabolic velocity profile, the specific pore residence time associated with either the inlet or outlet face is

$$
\tau_{i, j}=\bar{\tau}_{i, j} \frac{1}{2 \sqrt{1-x}},
$$

or, combining Eq. 14 with the notation used in the network equations,

$$
\tau_{i, j}=\frac{v_{i, j}}{\left|\left(p_{j}-p_{i}\right) g_{i, j}\right|} \frac{1}{2 \sqrt{1-x}} .
$$

In Eqs. 14 and 15, $x$ is a random variable chosen from a uniform distribution between zero and one. The origin of this term is from the probabilistic velocity argument. A detailed derivation of both $v_{i, j}$ and $\tau_{i, j}$. can be found in Thompson (1996).

From Eq. 15, the overall algorithm for simulating the RTD follows easily. Selection of the entrance throat is weighted in proportion to the fraction of the total injected flow rate. A tracer particle's interior path is determined from flow proportionality arguments, and as a particle passes through each pore, the time contributed to the overall residence time is calculated. A new value for the random parameter $x$ is found once per pore according to the assumption of mixing at the pore throat junctions.

The external RTD is found by simulating the preceding process for 10,000 tracer elements. To obtain a dimensional outlet concentration, the expected range of transit times is divided into a number of discrete intervals. During the injection process, the number of particles that exit during each interval is recorded, to be translated into an outlet concentration by determining the fraction of outlet volume represented by the tracer. The output data from this simulation are recorded as a series of points showing concentration vs. time, as one would obtain from a tracer experiment if the effluent is analyzed as discrete samples.

\section{Additional notes}

First, in the development of the network and the solution for single-phase flow, all stochastic information used to account for microscopic disorder is introduced in step 1: the points from which spheres are dropped into the bed are selected randomly from the cross section, and the diameter of the dropped spheres are selected randomly from a specified size distribution. The advantage of this procedure is that the disorder in the bed arises naturally from quantities that one would select for a real packed bed (bed dimensions, sphere sizes). This methodology is superior to introducing heterogeneity directly into the pore structure by way of the lattice arrangement and pore-throat-size distribution (both of which must be inferred secondarily).

Second, no restrictions exist concerning the morphology of the bed created in step 1 (it is limited only by the computer algorithm used). Hence, one can introduce heterogeneity of arbitrary scale and structure into step 2 and be assured that it is transferred into the network.

\section{Model Results}

Solution of the network equations provides the pressure in each pore of the packed bed, and hence the volumetric flow rate passing through each pore. This information, combined with known dimensional properties of the overall bed (length, porosity, cross-sectional area, etc.), is used to calculate averaged macroscopic parameters such as permeability. 
While the quantitative prediction of these parameters is an effective means to demonstrate the scale-up of microscopic information, the significance of this model is for use in situations where a medium's morphology or the quantity of interest precludes the use of empirical or other macroscopic relations. Applications may include the modeling and design of processes that depend strongly on pore-scale heterogeneity (e.g., dispersion and chromatography), analysis of processes where generalized empirical correlations fail, and the estimation of interfacial areas and mass-transfer coefficients in multiphase systems. The example below shows the prediction of macroscopic permeability. It is followed by three examples where a knowledge of microscale flow is required to reproduce flow patterns from actual disordered and heterogeneous beds.

\section{Macroscopic flow parameters}

Equation 7 makes all throat conductivities dimensional, so the inlet volumetric flow rate is calculated simply by summing the product of pressure drop and conductivity over all inlet faces in the bed:

$$
Q=\sum_{j=\text { inlet }}\left(p_{\text {inlet }}-p_{i}\right) g_{i, j}
$$

Successful setup and solution of the model equations will ensure that an outlet flow rate equal to the inlet flow rate is obtained using an analogous expression to Eq. 16. The bed dimensions used in the calculations are for the tessellated region only: the length of the bed is fixed by the limits imposed during the tessellation process, while the average cross-sectional area is found from Eq. 1 . Given $Q, p_{\text {inlet }}$, and the bed dimensions, the Darcy velocity, permeability, pressure gradient, space time, and Reynolds number can be calculated from standard formulas. For example, the average permeability is calculated from the simple form of Darcy's law for macroscopically one-dimensional (1-D) flow,

$$
K=\mu \frac{Q}{A_{c s}} \frac{L_{\text {bed }}}{\Delta p} .
$$

The direct use of Darcy's law to calculate a quantitative permeability is possible because the spatial dimensions of the packed bed are known (whereas, in a typical network of bonds and nodes, the relationship between volumetric flow rate and Darcy velocity is not well defined). Figure 6 is the program output showing predictions of a number of macroscopic quantities for a bed of nearly uniform $127-\mu \mathrm{m}$ spheres (std. dev. $=1.27 \mu \mathrm{m}$ ). The quantities are given in actual dimensions, and were obtained solely from the detailed description of the packed bed.

Because permeability is the most basic flow quantity used to study porous media, we investigate the permeability predictions from the model over a large range of sphere diameters (the beds for each point were generated independently). A comparison to experimental data as well as the Ergun equation (Bird et al., 1960, p. 200) is shown in Figure 7. The values for permeability are $30 \%$ below predictions by the $\mathrm{Er}$ -

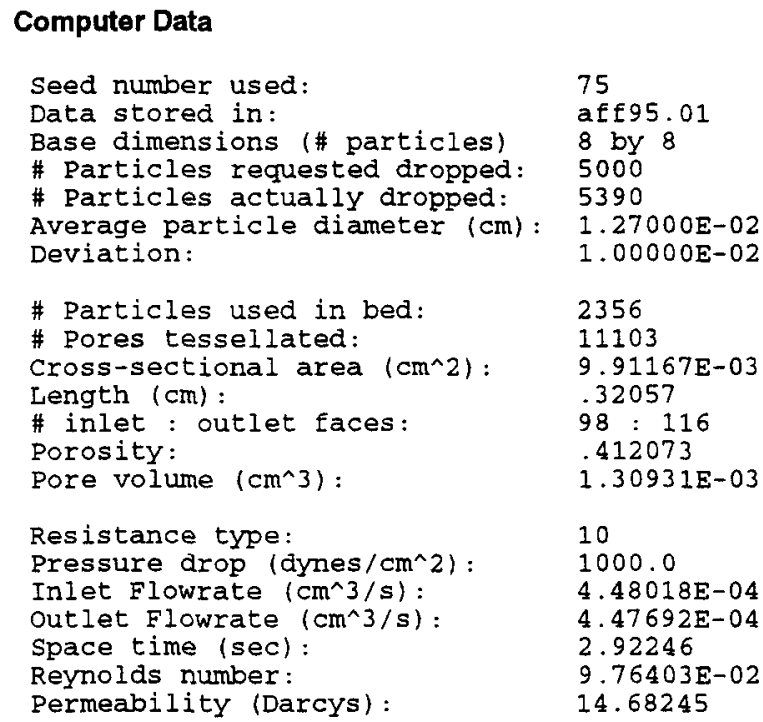

75

aff 95.01

8 by 8

5000

5390

1. $00000 \mathrm{E}-02$

2356

11103

$9.91167 \mathrm{E}-03$

.32057

$98: 116$

.412073

1. $30931 \mathrm{E}-03$

10

1000.0

$4.48018 \mathrm{E}-04$

$4.47692 \mathrm{E}-04$

2.92246

$9.76403 \mathrm{E}-02$

14.68245

\section{Experimental Data (Wyllie and Gregory, 1955)}

$\begin{array}{ll}\text { particle diameter (microns) } & 127 \\ \text { porosity } & 38-39 \% \\ \text { permeability (Darcys) } & 13.2\end{array}$

Figure 6. Results from packed bed model.

gun equation; taking the Ergun equation to be correct, one could make reasonable physical arguments to adjust $L$ in Eq. 12 so that the permeabilities match. At the present time we have chosen not to adjust any parameters in order to fit data. The values for permeability do fall within the fairly wide range of various experimental results (Wyllie and Gregory, 1955; Ahmed and Sunada, 1969), and the permeability increases as the square of particle size, which is correct.

\section{Direct simulation of experimental data}

If the simulated packings are essentially identical (within the bounds of natural disorder and minor physical constraints) in configuration to those used in experimental work, then the model output does not have to be scaled or be composed of intensive parameters. In fact, comparative quantities used to test the model can indeed be scale-dependent. In this section, the model is used to recreate an external resi-

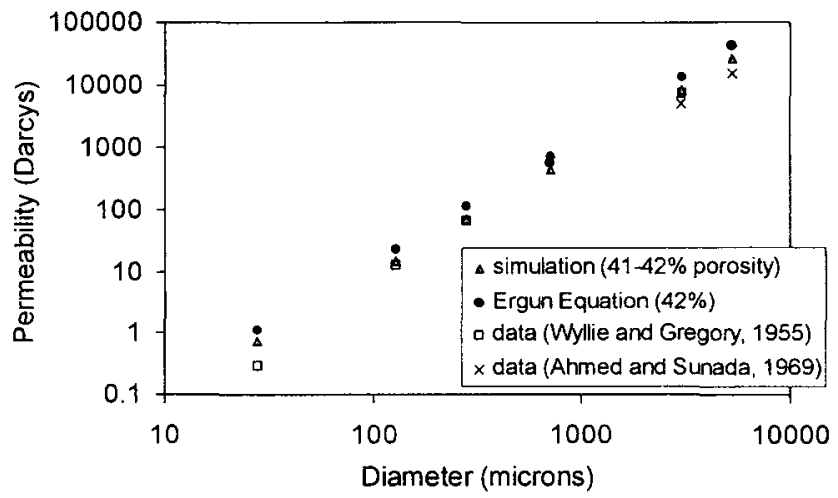

Figure 7. Prediction of permeability compared to Ergun equation and experiment. 
Table 1. Comparison of Real and Simulated Packed Beds

\begin{tabular}{lcc}
\hline & $\begin{array}{c}\text { Simulated } \\
\text { Bed }\end{array}$ & $\begin{array}{c}\text { Packed } \\
\text { Bed }\end{array}$ \\
\hline Particle diameter (cm) & 0.2 & 0.2 \\
No. particles in packed bed & 7,483 & $\sim 7,500$ \\
Cross-sectional area $\left(\mathrm{cm}^{2}\right)$ & 5.18 & 5.07 \\
Bed length $\left(\mathrm{cm}^{2}\right)$ & 8.35 & 10.7 \\
Pore volume $\left(\mathrm{cm}^{3}\right)$ & 17.85 & 24 \\
Pulse volume $(\%$ pore vol.) & 0.015 & 0.015 \\
Porosity (\%) & 41.3 & $41-44$ \\
Permeability (Darcy) & 4,126 & \\
\hline
\end{tabular}

dence-time distribution for a packed bed of uniform-sized glass spheres. RTDs are commonly used in reaction engineering to identify flow maldistribution and to quantify fluid residence times (Robinson and Tester, 1986). In most applications, the RTD is treated as a macroscopic parameter from which flow maldistribution can be evaluated using known correlations. However, an RTD originates at the pore level and is an excellent example demonstrating a scale-dependent macroscopic phenomenon.

The bed used for the experimental results was made with a 1-in.-ID (25.4-mm-ID) chromatography column and 0.2-cm glass spheres. Approximately 7,500 spheres were used, which provided a reasonably sized experimental bed while preventing the network problem from becoming overly laborious. Dimensions of the actual column vs. the simulated version are compared in Table 1. They are similar in most respects. The discrepancy in pore volume is attributed to a number of factors: slightly more spheres were probably used in the real packing; a slightly higher internal porosity (away from the walls) may have been obtained for the real packing; edge effects occur in the actual bed, thus increasing its overall porosity. The experimental setup did not allow accurate permeability measurement because of the very small pressure drop across the bed itself.

The experimental RTD and the model results are shown together in Figure 8. The agreement is good, including the sharp rise in concentration along with the extended tail and the presence of a single peak shifted slightly to the left of 1 PV. It should be noted that the use of pore volumes on the abscissa serves to align the curves each with their bed's respective pore volume, but does not mask other differences

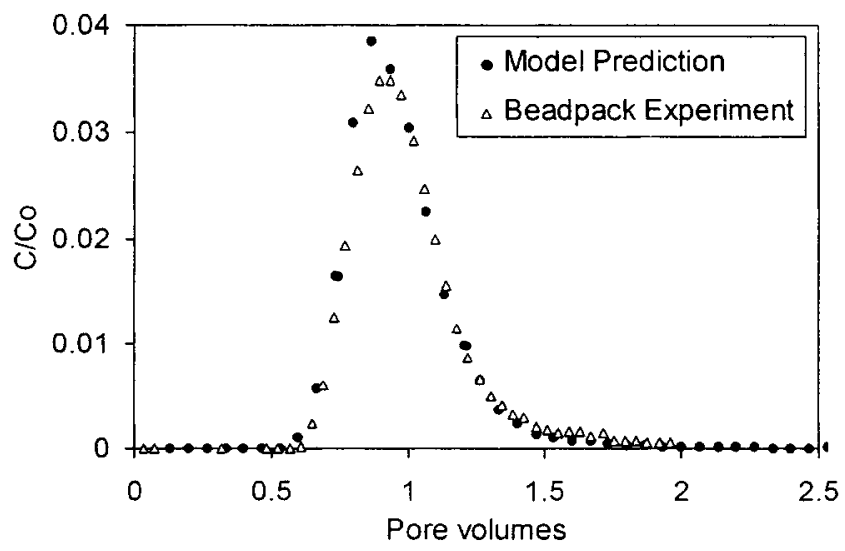

Figure 8. Residence time distribution in experimental vs. simulated packed beds. that may exist between the model and the actual RTD. In this regard, the slight difference between the curves is consistent with the actual bed being longer and having a slightly higher porosity. The model data shown in Figure 8 are significant for two reasons. Not only do they demonstrate the scale-up of dimensional macroscopic quantities from a knowledge of pore-scale flow, but they show that flow analysis on a complete packed bed can be performed using a network approach.

\section{Larger-scale heterogeneity and flow maldistribution}

Flow maldistribution is of concern in reaction engineering and separations processes because flow channeling and bypassing translate to low reactant conversions or insufficient contacting time. Although macroscopic correlations can often be used to predict the effects of flow maldistribution, the phenomenon originates at the pore scale where packing heterogeneity creates regions of high and low fluid conductivity. Connecting these pore-scale events to macroscopic responses requires a knowledge of the complex interactions within the interconnected pore space.

Oliveros and Smith (1982) created unique, artificially nonuniform packing arrangements to study flow maldistribution. These packed beds were each composed of a core region containing smaller spheres and an annular region containing larger spheres. They observed that, despite channeling through the higher permeability annulus, the RTDs from their beds had only one peak unless fluid exchange between the core and annular regions was prevented. The merge of two peaks into one (when communication was allowed) clearly illustrates how pore-scale flow patterns interact to affect the overall process. Oliveros and Smith (1982) give only one result that compares equivalent beds both with and without a barrier separating the annulus and core regions. The dimensions of their actual bed and the simulated version are given in Table 2. Although it is possible to model the entire bed, the simulated version was created to be approximately half the length of the actual in the interest of efficiency. When modeling the case where communication was allowed, the two simulated beds were placed end-to-end.

With a barrier present between the core and annulus, flow through each region is independent. The model simulations were performed by setting a constant pressure drop across

Table 2. Parameters Comparing Simulated Packed Bed to the Bed used by Oliveros and Smith (1992)

\begin{tabular}{lcc}
\hline & $\begin{array}{c}\text { Actual } \\
\text { Bed }\end{array}$ & $\begin{array}{c}\text { Simulated } \\
\text { Bed }\end{array}$ \\
\hline Core & & \\
Radius $(\mathrm{cm})$ & 2.91 & 2.87 \\
Cross-sectional area $\left(\mathrm{cm}^{2}\right)$ & 26.6 & 25.9 \\
Sphere diameter $(\mathrm{cm})$ & 0.5 & 0.5 \\
$\quad$ Porosity & 0.38 & 0.41 \\
Annulus & & \\
Width (cm) & 1.26 & 1.34 \\
Cross-sectional area $\left(\mathrm{cm}^{2}\right)$ & 28.0 & 29.7 \\
Sphere diameter $(\mathrm{cm})$ & 0.6 & 0.6 \\
$\quad$ Porosity & 0.45 & 0.45 \\
Bed & & \\
Length (cm) & 59.4 & 24.2 \\
Porosity & 0.42 & 0.43 \\
$\quad$ No. pores & & 58,108 \\
\hline
\end{tabular}


(A)

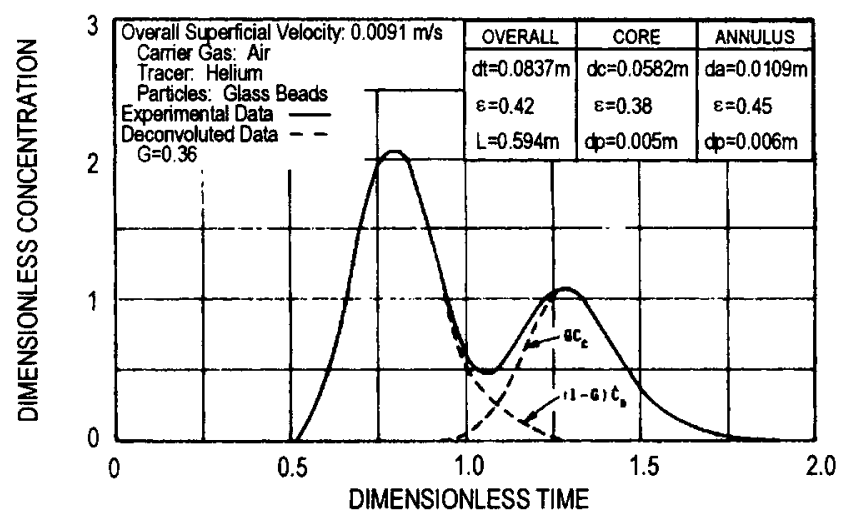

(B)

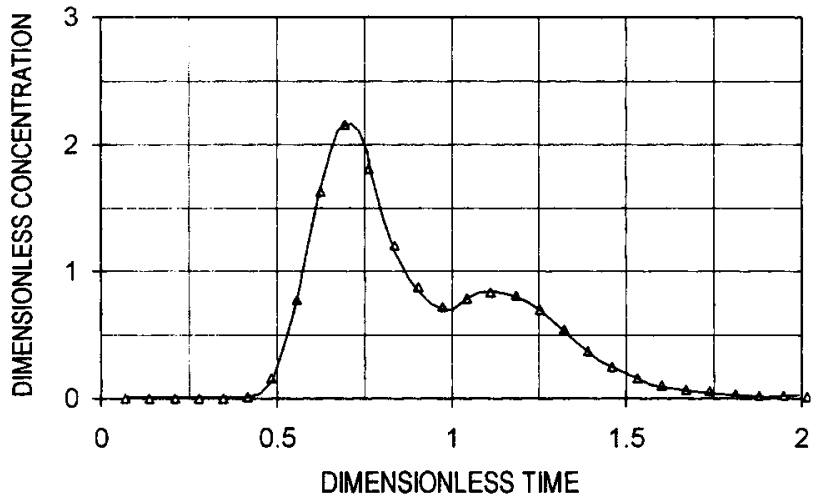

Figure 9. RTDs from heterogeneous bed with separation between zones.

(a) Experimental data reproduced from Oliveros and Smith (1982). (b) Model prediction.

the bed and sending a fraction of the tracer pulse through each region in proportion to its volumetric flow rate. Outlet tracer elements were combined to obtain the effluent concentration profile. The experimental results with the separating barrier are reproduced in Figure 9a, and data from the model are shown in Figure $9 b$.

The more interesting phenomenon occurs when the separation between the core and annulus is removed. Without the barrier, it is possible for slower-moving fluid elements in the core to migrate into more rapidly flowing streamlines in the annulus, and vice versa. The frequency of these migrations depends largely on the geometry of the system (i.e., the amount of fluid interchange between regions). Hence, it becomes apparent that any change from the RTD observed in Figure 9 could be predicted only with a knowledge of the pore flow patterns. The simulation without the barrier present is straightforward and performed as if the bed were homogeneous, which is possible because the fluid flow is based solely on the medium's microscopic structure. At this length scale, the presence of larger-scale heterogeneity cannot be detected, yet it is accounted for when the network equations are solved. The simulation shown in Figure 10b reveals that when interaction between the annular and core zones is allowed, the residence time distribution is a single-
(A)

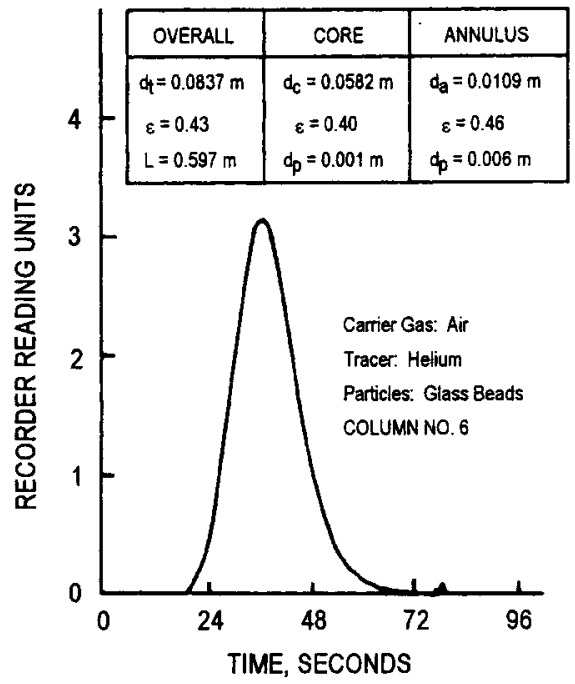

(B)

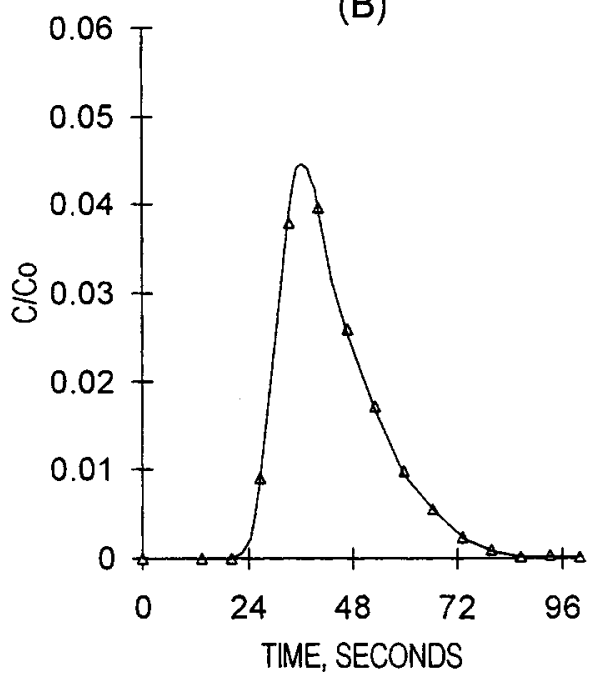

Figure 10. RTDs from heterogeneous bed with hydraulic communication between zones.

(a) Experimental data reproduced from Oliveros and Smith (1982). (b) Model prediction.

peaked function. This result agrees qualitatively with the experimental results reproduced in Figure 10a. (The axis units used and the information given in the referenced work precludes a quantitative comparison of the RTDs for the case with zone communication.)

\section{Dispersion and pore-scale heterogeneity}

Microscopic fluid velocities in a packed bed are highly variable because of the distribution of pore sizes and local effects from the bounding solid. Furthermore, the path length required for a solute molecule to traverse the bed varies because of the continual splitting and rejoining of streamlines. The aggregate effect of these phenomena causes a distribution of exit residence times and the dispersion of a solute pulse while it is in the medium. Network approaches provide 
much of the information needed to model the effects of convective dispersion, although it is more difficult to account for the effect of molecular diffusion on dispersion (diffusion allows solute molecules to move between slow and fast streamlines). This latter effect is particularly important at Peclet numbers less than 10 (Dullien, 1979), but also in cases where molecular diffusion can carry solute to regions of stagnation such as dead-end pores. Network models have been adapted to account for diffusivity (e.g., Sorbie and Clifford, 1991), but the inherent problem that cannot currently be addressed is that network models do not provide information about a solute's path through a flow junction (meaning streamlines lose their identity at these points). The probabilistic arguments that are used to assign direction and velocity out of a pore are equivalent to introducing an arbitrary diffusivity. In this article we examine the effects of pore structure on solute transport, using the assumption of complete fluid mixing in pores. Hence, the simulations may be viewed as representing a constant, but unknown Peclet number.

Nonuniform particle-size distribution is a common form of microscopic heterogeneity, yet its effects on phenomena such as dispersion are difficult to quantify in mathematical models. Han et al. (1985) performed an experimental investigation on the effects of particle size distribution and column length on dispersion. We use the packed bed model to reproduce three packings from their study: a uniform packed bed; distribution 1, which consists of four sizes of particles between $0.25 \mathrm{~cm}$ and $0.55 \mathrm{~cm}$, and distribution 2 , which consists of five particle sizes ranging from $0.25 \mathrm{~cm}$ to $1.58 \mathrm{~cm}$. All three packed beds are designed to have a mean particle diameter of $0.35 \mathrm{~cm}$. The experimental work demonstrates changes in longitudinal dispersion as measured at various axial positions in the bed up to a dimensionless length $L / d_{p} \approx$ 400 (the length over mean particle diameter). The values of $L / d_{p}$ for the computer-simulated beds ranged from 40 to 60 , so beds were placed in series to obtain dispersion coefficients at larger lengths. This procedure may have caused some anomalous effects as discussed below, but other options are not currently viable.

In the network model, dispersion coefficients were calculated in a manner similar to that described elsewhere (Sahimi et al., 1986; Sorbie and Clifford, 1991). Han et al. (1985) report dispersivity as a dimensionless ratio of dispersion coefficient to diffusion coefficient. The results presented here are for a dimensional dispersion coefficient because molecular diffusion is not included in the model for the reasons stated earlier. Figure 11 shows the dispersion coefficient as a function of the dimensionless bed length for the three packed beds. The results agree with the experimental work in the following aspects: The uniform bed and distribution 1 have similar dispersion coefficients that become constant well before $L / d_{p}=400$; the dispersion coefficient for distribution 2 is higher, and increases over the entire length range, never reaching a final value; the value of the predicted dispersion coefficients for the uniform bed and distribution 1 agree with the high-Peclet-number experiments $(P e \approx 3400)$ in the referenced work. We note, as described before, that the last comparison is not rigorous because an arbitrary diffusivity is introduced in network models by assuming complete mixing at nodes. The one significant difference between the model and the experimental work is the magnitude of the dispersion co-

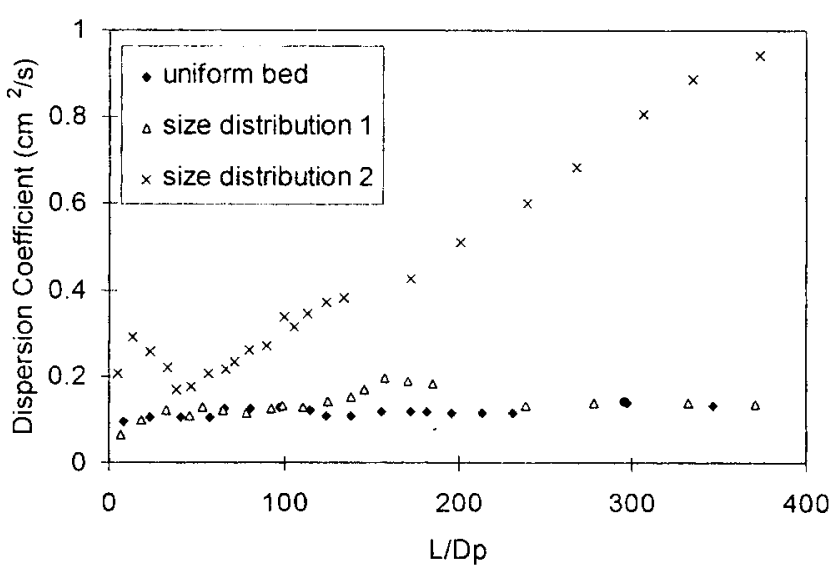

Figure 11. Dispersion coefficient as a function of bed length for uniform and nonuniform beds.

efficient for distribution 2, which is larger in the model. Experimentally, the dispersion coefficient for distribution 2 shows a monotonic increase over the range tested, but its magnitude is only slightly higher than for the other two beds. The discrepancy in the model result is probably a scaling problem, resulting from the bed length not being large enough to adequately represent the heterogeneity; the length of the repeated bed is 40 times average diameter, yet the large spheres are also large $(4.5 \times)$ relative to the mean. Hence, a few large spheres that lie together could cause a significant flow maldistribution, and while a large bed (i.e., a bed used in an experiment) would damp this heterogeneity out, the periodic nature of the model beds in series may instead magnify it. This issue illustrates problems to be addressed as porescale techniques become important for modeling larger-scale heterogeneity.

\section{Summary}

A model for flow through a disordered packed bed of spheres has been discussed. The modeling approach uses a network strategy, which takes advantage of the ability to incorporate microscopic heterogeneity into the structure. However, by including a rigorous description of the medium, the network structure arises directly from the medium's morphology rather than by inferences from a secondary interpretation of the pore-space geometry. Furthermore, the analysis of the pore-scale fluid mechanics provides a quantitative match with known or experimental macroscopic transport parameters.

The network originates from a complete description of a packed bed obtained from a computer simulation. Given the position and diameter of every sphere in the packed bed, a Delaunay tessellation is used to discretize the pore space into distinct tetrahedral elements representing individual pores. The tessellation also provides the interconnectivity of the system. From the microscale geometry within these discrete pore spaces, fluid conductivities are assigned by numerically solving the Navier-Stokes equations for flow through the porethroat cross sections. Finally, knowing the interconnectivity and the fluid conductivities in the tessellated network, the overall flow profile is solved using a network approach. 
The permeabilities for uniform beds of various sphere diameters fall within the bounds of experimental results, and without the use of adjustable parameters, permeability predictions are within $30 \%$ of empirical equations. A uniform bed used in experiments was modeled completely, to which scale-dependent quantities were compared. The external residence time was successfully modeled with excellent quantitative agreement. External RTDs from experiments on macroscopically heterogeneous packed beds were reproduced, and dispersion coefficients from nonuniform beds were calculated correctly if the scale of the bed was large enough compared to the scale of the heterogeneity. The important aspect of the model is its ability to incorporate arbitrary structural heterogeneity. Heterogeneity can be introduced at any length scale, and the pore-scale analysis of the fluid mechanics will remain correct. Scale-up to macroscopic flow using the network approach ensures that disorder at any length scale is included.

\section{Acknowledgments}

Funding for this work was provided by the Industrial Affiliates Program for Flow and Reaction in Porous Media at the University of Michigan: Aramco, ARCO, Chevron Petroleum Technology Co., Dowell Schlumberger, Conoco, Halliburton Services, Mobil, Texaco, and Unocal. We also thank Prof. Erik Thompson for providing the computer code for the finite-element simulations.

\section{Literature Cited}

Ahmed, N., and D. K. Sunada, "Nonlinear Flow in Porous Media," J. Hydraulics Div. ASCE, 95(HY6), 1847 (1969).

Bird, R. B., W. E. Stewart, and E. N. Lightfoot, Transport Phenomena, Wiley, New York (1960).

Blunt, M., and P. King, "Macroscopic Parameters from Simulations of Pore Scale Flow," Phys. Rev. A, 42(8), 4780 (1990).

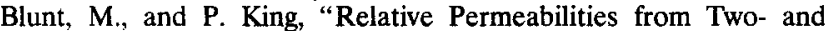
Three-Dimensional Pore-Scale Network Modelling," Transp. Porous Media, 6, 407 (1991).

Bowyer, A., "Computing Dirichlet Tessellations," Comp. J., 24(2), 162 (1981).

Bryant, S. L., D. W. Mellor, and C. A. Cade, "Physically Representative Network Models of Transport in Porous Media," AIChE J., 39(3), 387 (1993a).

Bryant, S. L., P. R. King, and D. W. Mellor, "Network Model Evaluation of Permeability and Spatial Correlation in a Real Random Sphere Packing," Transp. Porous Media, 11, 53 (1993b).

Bryant, S. L., and M. Blunt, "Prediction of Relative Permeability in Simple Porous Media," Phys. Rev. A, 46(4), 2004 (1992).

Chan, S. K., and K. M. Ng, "Geometrical Characteristics of the Pore Space in a Random Packing of Equal Spheres," Powder Technol., 54, 147 (1988).

Constantinides, G. N., and A. C. Payatakes, "A Three-Dimensional Network Model for Consolidated Porous Media. Basic Studies," Chem. Eng. Commun., 81, 55 (1989).

Dias, M. M., and A. C. Payatakes, "Network Models for Two-Phase Flow in Porous Media: 1. Immiscible Microdisplacement of Nonwetting Fluids," J. Fluid Mech., 164, 305 (1986).

Dullien, F. A. L., Porous Media: Fluid Transport and Pore Structure, Academic Press, New York (1979).

Dullien, F. A. L., "New Network Permeability Model of Porous Media," AIChE J., 21(2), 299 (1975).

Fatt, I., “The Network Model of Porous Media: I, II, III," Pet. Trans. AIME, 207, 144 (1956).

Han, N.-W., J. Bhakta, and R. G. Carbonell, "Longitudinal and Lateral Dispersion in Packed Beds: Effect of Column Length and Particle Size Distribution," AIChE J., 31(2), 277 (1985).

Happel, J., and H. Brenner, Low Reynolds Number Hydrodynamics, 2nd ed., Noordhoff, Leyden (1973).

Heiba, A. A., M. Sahimi, L. E. Scriven, and H. T. Davis, "Percolation Theory of Two-Phase Relative Permeability," SPE Res. Eng., 123 (1992).
Hoefner, M. L., and H. S. Fogler, "Pore Evolution and Channel Formation during Flow and Reaction in Porous Media," AIChE J., 34(1), 45 (1988).

Hopkins, M. R., and K. M. Ng, "Liquid-Liquid Relative Permeability, Network Models and Experiments," Chem. Eng. Commun., 46, 253 (1986).

Jerauld, G. R., J. C. Hatfield, L. E. Scriven, and H. T. Davis, "Percolation and Conduction on Voronoi and Triangular Networks: A Case Study in Topological Disorder," J. Phys. C. Solid State Phys., 17, 1519 (1984a).

Jerauld, G. R., L. E. Scriven, and H. T. Davis, "Percolation and Conduction on the 3DVoronoi and Regular Networks: A Second Case Study in Topological Disorder," J. Phys. C, 17, 3429 (1984b).

Kaviany, M., Principles of Heat Transfer in Porous Media, SpringerVerlag, New York (1991).

Koplik, J., C. Lin, and M. Vermette, "Conductivity and Permeability from Microgeometry," J. Appl. Phys., 56(11), 3127 (1984).

Koplik, J., "Creeping Flow in Two-Dimensional Networks," J. Fluid Mech., 119, 219 (1982).

Larson, R. G., L. E. Scriven, and H. T. Davis, "Percolation Theory of Two Phase Flow in Porous Media," Chem. Eng. Sci., 36, 57 (1981).

Lin, C.-Y., and J. C. Slattery, "Three-Dimensional, Randomized Network Model for Two-Phase Flow Through Porous Media," AIChE J., 28, 311 (1982).

Mason, G., and D. W. Mellor, "Analysis of the Percolation Properties of a Real Porous Medium," Characterization of Porous Solids II, F. Rodriquez-Reinoso et al., eds., Elsevier, Amsterdam, p. 41 (1991).

Mason, G., General Discussions, Discuss. Farad. Soc., 43, 75 (1967).

Mellor, D. W., "Random Close Packing (RCP) of Equal Spheres: Structure and Implications for Use as a Model Porous Medium," PhD Thesis, Open Univ., Milton Keynes, England (1989).

Mohanty, K. K., and S. J. Salter, "Multiphase Flow in Porous Media: II. Pore-level Modeling," Tech. Conf. of the SPE-AIME, New Orleans, LA (1982).

Oliveros, G., and J. M. Smith, "Dynamic Studies of Dispersion and Channeling in Fixed Beds," AIChE J., 28(5), 751 (1982).

Rege, S. D., and H. S. Fogler, "A Network Model for Deep Bed Filtration of Solid Particles and Emulsion Drops," AIChEJ., 34(11), 1761 (1988).

Rege, S. D., and H. S. Fogler, "Network Model for Straining Dominated Particle Entrapment in Porous Media," Chem. Eng. Sci., 42(7), 1553 (1987).

Robinson, B. A., and J. W. Tester, "Characterization of Flow Maldistribution using Inlet-Outlet Tracer Techniques: An Application of Internal Residence Time Distributions," Chem. Eng. Sci., 41(3), 469 (1986).

Sahimi, M., B. D. Hughes, L. E. Scriven, and H. T. Davis, "Dispersion in Flow Through Porous Media-I. One-Phase Flow," Chem. Eng. Sci., 41(8), 2103 (1986).

Sahimi, M., H. T. Davis, and L. E. Scriven, "Dispersion in Disordered Porous Media," Chem. Eng. Commun., 23, 329 (1983).

Sorbie, K. S., and P. J. Clifford, "The Inclusion of Molecular Diffusion Effects in the Network Modelling of Hydrodynamic Dispersion in Porous Media," Chem. Eng. Sci., 46(10), 2525 (1991).

Thompson, K. E., "Interfacial Reactions for the Modification of Flow in Porous Media," PhD Thesis, Univ. of Michigan, Ann Arbor (1996).

Visscher, W. M., and M. Bolsterli, "Random Packing of Equal and Unequal Spheres in Two and Three Dimensions," Nature, 239, 505 (1972).

Watson, D. F., "Computing the n-Dimensional Delaunay Tessellation with Application to Voronoi Polytopes," Comp. J., 24(2), 167 (1981).

Wyllie, M. R. J., and A. R. Gregory, "Fluid Flow through Unconsolidated Porous Aggregates," Ind. Eng. Chem., 47(7), 1379 (1955).

Yang, A., C. T. Miller, and L. D. Turcoliver, "Simulation of Correlated and Uncorrelated Packing of Random Size Spheres," Phys. Rev. E, 53(2), 1516 (1996).

Yilmaz, T. "General Equations for Pressure Drop for Laminar Flow in Ducts of Arbitrary Cross Sections," J. Energy Res. Tech., 112, 220 (1990).

Manuscript received May 29, 1996, and revision received Jan. 22, 1997. 